\title{
Ab interno trabeculectomy: patient selection and perspectives
}

\author{
This article was published in the following Dove Press journal: \\ Clinical Ophthalmology \\ 17 August 2016 \\ Number of times this article has been viewed
}

\section{Kateki Vinod \\ Steven J Gedde}

Bascom Palmer Eye Institute, University of Miami Miller School of Medicine, Miami, FL, USA
Correspondence: Steven J Gedde

Bascom Palmer Eye Institute, University of Miami Miller School of Medicine, 900 NW 17th Street, Miami, FL 33।36, USA

Email sgedde@med.miami.edu

\begin{abstract}
Ab}$ interno trabeculectomy is one among several recently introduced minimally invasive glaucoma surgeries that avoid a conjunctival incision and full-thickness sclerostomy involved in traditional glaucoma surgery. Ablation of the trabecular meshwork and inner wall of Schlemm's canal is performed in an arcuate fashion via a clear corneal incision, alone or in combination with phacoemulsification cataract surgery. Intraocular pressure reduction following ab interno trabeculectomy is limited by resistance in distal outflow pathways and generally stabilizes in the mid-to-high teens. Relief of medication burden has been demonstrated by some studies. A very low rate of complications, most commonly transient hyphema and intraocular pressure elevations in the immediate postoperative period, have been reported. However, available data are derived from small retrospective and prospective case series. Randomized, controlled trials are needed to better elucidate the potential merits of ab interno trabeculectomy in the combined setting versus phacoemulsification cataract surgery alone and to compare it with other minimally invasive glaucoma surgeries.
\end{abstract}

Keywords: ab interno trabeculectomy, glaucoma, intraocular pressure, minimally invasive glaucoma surgery, surgical outcomes

\section{Introduction}

The surgical management of glaucoma has evolved in recent years to include a number of conjunctival-sparing procedures termed minimally- or microinvasive glaucoma surgery (MIGS). ${ }^{1}$ As the designation suggests, MIGS offers an improved safety profile when compared with traditional incisional glaucoma surgery (trabeculectomy with or without antifibrotics and tube shunt surgery), albeit at the expense of a smaller reduction in intraocular pressure (IOP).

$\mathrm{Ab}$ interno trabeculectomy (AIT) was among the earliest MIGS procedures introduced into the armamentarium of surgical glaucoma therapies. Since receiving approval by the United States Food and Drug Administration (FDA) in 2004, AIT has been evaluated in prospective case series and small retrospective studies but not in a randomized controlled trial. This review summarizes the safety and efficacy of AIT using data from prospective case series, and discusses the clinical applications of the procedure.

\section{Device and surgical approach}

The device used to perform AIT is marketed as the Trabectome by NeoMedix, Inc (Tustin, CA, USA). It consists of a $19.5 \mathrm{G}$ hand piece with a bipolar $550 \mathrm{kHz}$ electrode tip containing irrigation and aspiration ports, which are controlled by a foot pedal. After positioning the patient's head and operating microscope to facilitate visualization of the nasal angle with a gonioscopy lens, the electrode tip is introduced into the anterior 
chamber via a 1.6-1.8 mm temporal clear corneal incision and used to engage the trabecular meshwork (TM). A strip of TM and the inner wall of Schlemm's canal are then ablated by advancing the device tip in a clockwise or counterclockwise direction for a total of $30^{\circ}-180^{\circ}$. An opening in Schlemm's canal is created that permits direct aqueous egress into the collector channels. An initial power of $0.7 \mathrm{~mW}$ can be increased to achieve a wider ablation zone or decreased if coagulation is noted to occur. Remnants of ablated tissue are removed via aspiration. Viscoelastic may be injected into the anterior chamber over the arc of ablated tissue to minimize hyphema. Topical antibiotics, corticosteroids, and miotics are used postoperatively. When used as a combined procedure, AIT is generally performed first while the gonioscopic view is most optimal, and the clear corneal incision is subsequently enlarged for phacoemulsification cataract surgery. The larger corneal wound required for phacoemulsification can produce anterior chamber instability if AIT is performed secondarily. ${ }^{2-5}$ Ablation is achieved using plasma-mediated ionization and disintegration rather than electrocautery, which confines the dissipation of heat from the device tip to the treatment area. Histopathologic studies of cadaveric human eyes have provided evidence for the focal removal of TM and inner wall of Schlemm's canal without damage to the outer wall of Schlemm's canal or sclera. ${ }^{6}$

Proposed advantages of AIT include minimal thermal damage to adjacent tissues, lack of a full-thickness sclerostomy, and potential to be combined with phacoemulsification cataract surgery. Disadvantages include an inability to achieve very low IOP due to downstream resistance to flow (as is true for any MIGS that targets the TM) and possible eventual cleft closure.

\section{Efficacy}

\section{AIT alone}

Data from prospective case series evaluating outcomes after AIT as a standalone procedure are summarized in Table 1.
Minckler et $\mathrm{al}^{7}$ reported a reduction in mean $\pm \mathrm{SD}$ IOP from a baseline of $27.6 \pm 7.2 \mathrm{mmHg}(\mathrm{n}=101)$ to $16.3 \pm 3.3 \mathrm{mmHg}$ $(n=11) 30$ months after AIT in patients with open-angle glaucoma. Success (ie, IOP $\leq 21 \mathrm{mmHg}$ with or without medications and without additional glaucoma surgery) was $84 \%$ at 30 months. Maeda et $\mathrm{al}^{8}$ showed a reduction in mean $\pm \mathrm{SD}$ IOP and number of medications from $26.6 \pm 8.1 \mathrm{mmHg}$ and $4.0 \pm 1.4(\mathrm{n}=80)$ preoperatively to $17.9 \pm 6.1 \mathrm{mmHg}$ and $2.3 \pm 1.2(\mathrm{n}=27)$, respectively, among Japanese patients with open-angle glaucoma at 1 year. Similar IOP reduction to the mid-teens was found among 92 patients in Germany 1 year after AIT. ${ }^{9}$ In a prospective case series of pseudophakic Chinese patients with open-angle glaucoma, mean $\pm \mathrm{SD}$ preoperative IOP was $24.4 \pm 4.4 \mathrm{mmHg}$ using $3.9 \pm 0.8$ medications $(n=19)$ and was reduced to $15.9 \pm 5.1 \mathrm{mmHg}$ using $2.8 \pm 1.6$ medications ( $\mathrm{n}$, not reported). Overall success (ie, IOP $\leq 21 \mathrm{mmHg}$ with or without glaucoma medications and without additional glaucoma surgery) was $89.5 \%$ at 6 months postoperatively. ${ }^{10}$

In a study comparing outcomes after AIT alone in patients with primary open-angle glaucoma (POAG) and pseudoexfoliation glaucoma $(\mathrm{PXG})$, mean \pm SD IOP was reduced from $25.5 \pm 7.9 \mathrm{mmHg}(\mathrm{n}=450)$ to $16.8 \pm 3.9 \mathrm{mmHg}$ $(\mathrm{n}=293)$ and from $29.0 \pm 7.5 \mathrm{mmHg}(\mathrm{n}=67)$ to $16.1 \pm 4.0 \mathrm{mmHg}$ $(\mathrm{n}=53)$ at 1 year, respectively. Mean \pm SD IOP decrease was significantly greater in the PXG group $(-7.5 \pm 7.4 \mathrm{mmHg}$ in the POAG group versus $-12.3 \pm 8.0 \mathrm{mmHg}$ in the PXG group, $P<0.01)$, but this group had significantly higher baseline IOP $(P<0.01)$. Mean \pm SD number of medications was reduced from $2.73 \pm 1.33$ to $2.16 \pm 1.29$ in the POAG group and from $3.09 \pm 1.15$ to $2.21 \pm 1.38$ in the PXG group at 1 year. Cumulative success (ie, IOP $<21 \mathrm{mmHg}$ and $>20 \%$ reduction from baseline) was $62.9 \%$ and $79.1 \%$ in the POAG and PXG groups $(P=0.004)$, respectively, with a significantly greater need for additional glaucoma surgery in the POAG group $(P=0.02)$. This study used data voluntarily provided to the Trabectome Study Group database by surgeons from

Table I Summary of prospective case series evaluating outcomes after ab interno trabeculectomy alone

\begin{tabular}{|c|c|c|c|c|c|c|c|}
\hline \multirow[t]{2}{*}{ Author (year) } & \multirow[t]{2}{*}{$\mathbf{N}$} & \multicolumn{2}{|l|}{ IOP, $\mathrm{mmHg}$} & \multicolumn{2}{|c|}{ Number of medications } & \multirow{2}{*}{$\begin{array}{l}\text { Success } \\
\text { rate, } \%\end{array}$} & \multirow{2}{*}{$\begin{array}{l}\text { Mean follow- } \\
\text { up, months }\end{array}$} \\
\hline & & Preoperative & Postoperative & Preoperative & Postoperative & & \\
\hline Minckler et $\mathrm{al}^{7}$ (2006) & 101 & $27.6 \pm 7.2$ & $16.3 \pm 3.3$ & - & - & 84.0 & 30 \\
\hline \multirow[t]{2}{*}{ Ting et $\mathrm{al}^{\prime \prime}(2012)$} & 450 (POAG) & $25.5 \pm 7.9$ & $16.8 \pm 3.9$ & $2.73 \pm 1.33$ & $2.16 \pm 1.29$ & 62.9 & 12 \\
\hline & 67 (PXG) & $29.0 \pm 7.5$ & $16.1 \pm 4.0$ & $3.09 \pm 1.15$ & $2.21 \pm 1.38$ & 79.1 & \\
\hline Maeda et $\mathrm{al}^{8}(20 \mid 3)$ & 80 & $26.6 \pm 8.1$ & $17.9 \pm 6.1$ & $4.0 \pm 1.4$ & $2.3 \pm 1.2$ & - & 12 \\
\hline Werth et $\mathrm{al}^{9}(2015)$ & 92 & $20.0 \pm 7.0$ & $15.4 \pm 4.0$ & - & - & - & 12 \\
\hline \multirow[t]{2}{*}{ Mizoguchi et al ${ }^{12}$ (20l5) } & 43 (POAG) & $23.5 \pm 7.2$ & $\mid 4.1 \pm 2.2$ & $2.8 \pm 0.8$ & $1.8 \pm 1.0$ & 50.9 & 24 \\
\hline & 39 (PXG) & $21.7 \pm 6.2$ & $13.9 \pm 4.7$ & $2.7 \pm 0.8$ & $2.9 \pm 0.7$ & 49.2 & \\
\hline Lee et $\mathrm{al}^{10}(2016)$ & 19 & $24.4 \pm 4.4$ & $15.9 \pm 5.1$ & $3.9 \pm 0.8$ & $2.8 \pm 1.6$ & 89.5 & 6 \\
\hline
\end{tabular}

Notes: IOP and number of medications are reported as mean \pm SD. The dashes indicate that the data were not reported.

Abbreviations: IOP, intraocular pressure; POAG, primary open angle glaucoma; PXG, pseudoexfoliation glaucoma; SD, standard deviation. 
their first 20 AIT cases. ${ }^{11}$ Mizoguchi et al ${ }^{12}$ also compared eyes with POAG and PXG and found a mean \pm SD IOP reduction from $23.5 \pm 7.2 \mathrm{mmHg}(\mathrm{n}=43)$ to $14.1 \pm 2.2 \mathrm{mmHg}$ $(\mathrm{n}=8)$ in the POAG group and from $21.7 \pm 6.2 \mathrm{mmHg}(\mathrm{n}=39)$ to $13.9 \pm 4.7 \mathrm{mmHg}(\mathrm{n}=14)$ in the PXG group at 2 years. Mean \pm SD number of medications was reduced from $2.8 \pm 0.8$ to $1.8 \pm 1.0$ in the POAG group, while mean $\pm \mathrm{SD}$ number of medications was $2.7 \pm 0.8$ preoperatively and $2.9 \pm 0.7$ at 2 years in the PXG group. Success (ie, IOP $<21 \mathrm{mmHg}$ and $\geq 20 \%$ reduction from baseline without additional glaucoma surgery or need for an increased number of medications postoperatively) was comparable between the two groups at 2 years $(50.9 \%$ in the POAG group versus $49.2 \%$ in the PXG group, $P=0.91$ ) and lower than that reported in prior studies. ${ }^{12}$ A recent meta-analysis by Kaplowitz et al ${ }^{13}$ demonstrated a mean decrease of $10.5 \pm 1.9 \mathrm{mmHg}$ from a mean baseline IOP of $26.71 \pm 1.34 \mathrm{mmHg}$ using $0.99 \pm 0.54$ fewer glaucoma medications. Overall success (ie, IOP $\leq 21 \mathrm{mmHg}$ with a $20 \%$ reduction from baseline and without additional glaucoma surgery) was $46 \% \pm 34 \%$ at 2 years. There was a $9.77 \mathrm{mmHg}$ (95\% CI 8.90-10.64) weighted mean difference in IOP from baseline to study end point. As expected, differences in factors such as sample size, glaucoma type, follow-up, baseline IOP, and definition of success contributed to high heterogeneity in this study. ${ }^{13}$

In summary, AIT alone results in IOP lowering to the mid-to-high teens on average, but with a substantial drop-off in success rates with longer follow-up. Relief of medication burden postoperatively has been variable in published prospective series.

\section{AIT combined with phacoemulsification cataract surgery}

As with other MIGS procedures, AIT can be performed in conjunction with phacoemulsification cataract surgery (AIT-phaco) using the same clear corneal incision. Comparable visual and refractive outcomes were observed in a retrospective study of patients who underwent AIT-phaco versus phacoemulsification alone, without a significant increase in the risk of complications. ${ }^{14}$

Data from prospective case series evaluating outcomes after AIT-phaco are summarized in Table 2. In the metaanalysis by Kaplowitz et al, ${ }^{13}$ eyes that underwent AIT-phaco experienced a mean $\pm \mathrm{SD}$ IOP reduction of $6.24 \pm 1.98 \mathrm{mmHg}$ from a baseline IOP of $21 \pm 1.31 \mathrm{mmHg}$ using $0.76 \pm 0.35$ fewer glaucoma medications. The success rate was $85 \% \pm 7 \%$ at 2 years, compared with $46 \% \pm 34 \%$ for AIT alone. There was a $6.04 \mathrm{mmHg}(95 \%$ CI 4.95-7.13) weighted difference in mean IOP from baseline to study end point. Heterogeneity was high as noted earlier. ${ }^{13}$

Using data from the Trabectome Study Group database, Francis et $\mathrm{al}^{15}$ showed a reduction in mean IOP from $20.0 \pm 6.3 \mathrm{mmHg}$ at baseline $(\mathrm{n}=304)$ to $15.5 \pm 2.9 \mathrm{mmHg}$ $(n=34)$ at 1 year after AIT-phaco, with a concomitant reduction in mean \pm SD number of medications from $2.65 \pm 1.13$ at baseline to $1.44 \pm 1.29$ at 1 year. Success (ie, $\geq 20 \%$ decrease in mean IOP or mean medications without additional glaucoma laser or surgery) was $64 \%$ at 1 year. ${ }^{15}$

Only one case series has prospectively compared outcomes following AIT-phaco to phacoemulsification alone. Mean \pm SD IOP was reduced from $22.2 \pm 5.9 \mathrm{mmHg}(\mathrm{n}=114)$ to $15.3 \pm 3.5 \mathrm{mmHg}(\mathrm{n}=67)$ at 2 years after AIT-phaco, while mean \pm SD IOP was reduced from $16.2 \pm 4.2 \mathrm{mmHg}(\mathrm{n}=145)$ to $14.3 \pm 3.6 \mathrm{mmHg}(\mathrm{n}=41)$ at 2 years after phacoemulsification alone. The number of glaucoma medications was reduced by $40 \%$ from baseline to 2 years in the AIT-phaco group (mean values not reported). Cumulative success (ie, IOP $<21 \mathrm{mmHg}$ and $20 \%$ reduction from baseline without additional glaucoma surgery) was $80 \%$ in the combined group and $45 \%$ for the phacoemulsification alone group. ${ }^{16}$

Another prospective case series compared outcomes following AIT-phaco with those of trabeculectomy combined with phacoemulsification (phaco-trab). At 1 year, IOP was reduced from a baseline of $22.1 \pm 5.5 \mathrm{mmHg}$

Table 2 Summary of prospective case series evaluating outcomes after ab interno trabeculectomy combined with phacoemulsification

\begin{tabular}{|c|c|c|c|c|c|c|c|}
\hline \multirow[t]{2}{*}{ Author (year) } & \multirow[t]{2}{*}{$\mathbf{N}$} & \multicolumn{2}{|l|}{ IOP, mmHg } & \multicolumn{2}{|c|}{ Number of medications } & \multirow{2}{*}{$\begin{array}{l}\text { Success } \\
\text { rate, } \%\end{array}$} & \multirow{2}{*}{$\begin{array}{l}\text { Mean follow- } \\
\text { up, months }\end{array}$} \\
\hline & & Preoperative & Postoperative & Preoperative & Postoperative & & \\
\hline Francis et al ${ }^{15}$ (2008) & 304 & $20.0 \pm 6.3$ & $15.5 \pm 2.9$ & $2.65 \pm 1.13$ & $1.44 \pm 1.29$ & 64.0 & 12 \\
\hline Francis $^{16}(2010)$ & 114 & $22.2 \pm 5.9$ & $15.3 \pm 3.5$ & - & - & 80.0 & 24 \\
\hline Francis et al ${ }^{17}(201 \mathrm{I})$ & 89 & $22.1 \pm 5.5$ & $15.4 \pm 3.1$ & - & - & 95.0 & 12 \\
\hline \multirow[t]{2}{*}{ Ting et al" (20I2) } & 263 (POAG) & $19.9 \pm 5.4$ & $15.6 \pm 3.2$ & $2.40 \pm 1.08$ & $1.65 \pm 1.26$ & 91.0 & 12 \\
\hline & $45(\mathrm{PXG})$ & $21.7 \pm 8.4$ & $14.2 \pm 3.1$ & $2.53 \pm 0.99$ & $1.57 \pm 1.33$ & 86.7 & \\
\hline Werth et $\mathrm{al}^{9}$ (20।5) & 30 & $20.8 \pm 7.4$ & $16.4 \pm 5.5$ & - & - & - & 12 \\
\hline
\end{tabular}

Notes: IOP and number of medications are reported as mean \pm SD. The dashes indicate that the data were not reported.

Abbreviations: IOP, intraocular pressure; POAG, primary open angle glaucoma; PXG, pseudoexfoliation glaucoma; SD, standard deviation. 
$(n=89)$ to $15.4 \pm 3.1 \mathrm{mmHg}(\mathrm{n}=85)$ among patients in the AIT-phaco group, and from a baseline of $23.0 \pm 10.7 \mathrm{mmHg}$ $(n=23)$ to $11.0 \pm 5.7 \mathrm{mmHg}(n=20)$ in the phaco-trab group. Phaco-trab achieved a lower mean IOP at 1 year, but with an increased incidence of sustained hypotony 1 month postoperatively $(P<0.01)$. Cumulative success (ie, IOP $<21 \mathrm{mmHg}$ and $20 \%$ reduction from baseline without additional glaucoma surgery) was comparable at 1 year between the two groups, with AIT-phaco patients achieving 95\% success and phaco-trab patients achieving $83 \%$ success at 1 year $(P=0.1) .{ }^{17}$

Data from the Trabectome Study Group database were used to compare outcomes between patients with POAG $(n=263)$ and PXG $(n=45)$ after AIT-phaco. Mean \pm SD IOP was reduced from a baseline of $19.9 \pm 5.4 \mathrm{mmHg}$ to $15.6 \pm 3.2 \mathrm{mmHg}$ at 1 year in eyes with POAG $(\mathrm{n}=247)$ and from a baseline of $21.7 \pm 8.4 \mathrm{mmHg}$ to $14.2 \pm 3.1 \mathrm{mmHg}$ at 1 year in eyes with PXG $(n=42)$. Mean \pm SD number of glaucoma medications was reduced from a baseline of $2.40 \pm 1.08$ to $1.65 \pm 1.26$ in the POAG eyes and from a baseline of $2.53 \pm 0.99$ to $1.57 \pm 1.33$ in the PXG eyes at 1 year. The two groups had comparable success (ie, IOP $<21 \mathrm{mmHg}$ and $>20 \%$ reduction from baseline) of $91.0 \%$ in the POAG group and $86.7 \%$ in the PXG group $(P=0.73)$ at 1 year. In this study, success rates were higher in the AIT-phaco group than the AIT alone group for both POAG and PXG patients, possibly due to anterior chamber deepening after lens removal with resulting increase in outflow, and the need for supplemental medications was less. ${ }^{11}$ Werth et al ${ }^{9}$ found similar IOP lowering to the mid-teens after AIT-phaco.

Using an algorithm to match patients based on age, race, glaucoma subtype, baseline IOP, and number of medications, Parikh et al ${ }^{18}$ compared 255 phakic eyes that underwent AIT alone with 498 eyes that underwent AIT-phaco and found that phacoemulsification did not contribute to IOP lowering to a statistically significant degree $(P \geq 0.05)$. In short, results of prospective case series examining AIT-phaco are variable. A combined approach is generally associated with consistent IOP reduction to the mid-teens and fairly high success rates $(>80 \%)$, albeit with limited follow-up.

\section{Failure and reoperations}

Improper surgical technique can lead to failure following AIT with or without phacoemulsification. The use of high power can produce coagulation, inciting a wound healing process that can result in treatment failure. Incomplete removal of TM may allow for reapproximation of its severed ends from subsequent fibrosis. ${ }^{6}$ Fellman et al ${ }^{19}$ proposed the episcleral venous fluid wave (EVFW) as a means of intraoperatively assessing the patency of the trabecular outflow pathway. In a study of 68 eyes that underwent AIT with or without phacoemulsification, eyes with a prominent intraoperative EVFW suggestive of adequate flow through collector channels achieved a lower IOP with fewer medications than eyes without an intraoperative $\operatorname{EVFW}(P=0.001)$.

A retrospective study by Jea et $\mathrm{al}^{20}$ found lower baseline IOP and younger age to be additional risk factors for failure. Lower baseline IOP may be associated with higher rates of failure whenever surgical success is defined based on a percentage IOP reduction from baseline, since IOP cannot be lowered below episcleral venous pressure by AIT.

Reoperation rates following AIT of up to $35 \%$ have been reported..$^{7-13,15-17,20}$ A retrospective study by Jea et al ${ }^{21}$ comparing outcomes of subsequent trabeculectomy after failed AIT to primary trabeculectomy showed that failed AIT did not affect success rates.

\section{Safety}

Complications associated with trabeculectomy including bleb dysesthesia, bleb leak, blebitis, and endophthalmitis, as well as tube-related complications such as tube erosion, migration, and corneal decompensation, have been shown to occur at a low but significant rate in several large clinical trials. ${ }^{22-24}$ Minimally invasive glaucoma surgery tends to avoid these serious complications due to a "blebless" approach that uses an ab interno microincision rather than a full-thickness sclerostomy. ${ }^{1}$ AIT has been associated with non-sight threatening complications such as corneal epithelial defect, Descemet's membrane injury, iris trauma, anterior lens capsular damage, hyphema, peripheral anterior synechiae, and IOP "spikes", or sudden elevations. ${ }^{7-13,15-17,20}$ The rate of sight-threatening complications following AIT or AIT-phaco was $<1 \%$ in the meta-analysis by Kaplowitz et al. ${ }^{13}$ Hyphema was the most common complication followed by peripheral anterior synechiae and IOP spikes. ${ }^{13}$

Intraoperative blood reflux from Schlemm's canal is common and expected during AIT. Hyphema has been reported to occur in up to $70 \%-100 \%$ of eyes in the immediate postoperative period. ${ }^{13}$ Hyphema tends to be self-limited and has not been shown to contribute significantly to immediate postoperative IOP spikes. ${ }^{25}$ Ahuja et al ${ }^{26}$ described delayed-onset symptomatic hyphema in 12 of $262(4.6 \%)$ patients $>2$ months (range, 2-31 months) after AIT. One patient required subsequent trabeculectomy for IOP elevation relating to hyphema, while the remaining patients had spontaneous resolution of hyphema within 1-2 weeks. Sleeping on 
the side of the operated eye and exertion were suggested as possible risk factors. ${ }^{26}$ Delayed-onset hyphema has also been reported to occur during subsequent intraocular operations (in one case, during trabeculectomy performed 11 months after failed AIT, and in a second case, during phacoemulsification cataract surgery 2 years after failed AIT). ${ }^{27,28}$ Possible mechanisms include blood reflux from Schlemm's canal, focal ingrowth of episcleral vessels, membrane formation, and long-term sensitization of ablated tissue. In another case described by Mansouri et al, ${ }^{29}$ nocturnal IOP spikes were identified using a 24-hour contact lens IOP monitor in a patient who developed nighttime pain 11 months after AIT. Nocturnal hyphema was suggested as a mechanism for the symptoms, which resolved with a combination of prostaglandin and pilocarpine. ${ }^{29}$

Peripheral anterior synechiae and transient IOP spikes $\geq 10 \mathrm{mmHg}$ above baseline have been reported to occur in up to $24 \%$ of patients ${ }^{30}$ and in $4 \%-10 \%$ of patients, ${ }^{11,31}$ respectively, after AIT. Transient hypotony on the first postoperative day has been reported to occur infrequently and without visual sequelae. ${ }^{7,11,14}$ Transient ciliochoroidal detachments were detected using anterior-segment optical coherence tomography (AS-OCT) in 14 of 33 (42\%) eyes at the third postoperative day in a prospective observational study from Japan. Eyes with ciliochoroidal detachment had lower mean IOP than eyes that did not, but IOP was never $<5 \mathrm{mmHg}$ in any eyes. Only four eyes had persistent ciliochoroidal detachment at 1 month, but visual acuity was not reported. ${ }^{32}$ Osman and AlMobarak described a case of cyclodialysis cleft following AIT with hypotony (IOP $<5 \mathrm{mmHg}$ ), persisting to the fourth postoperative month at the time of the report. ${ }^{33}$ Rare cases of serious complications, such as aqueous misdirection, suprachoroidal hemorrhage, and endophthalmitis, have also been described. ${ }^{4,13,25,34,35}$

Two studies have compared outcomes following AIT to trabeculectomy with mitomycin $\mathrm{C}$. In a retrospective cohort study, Jea et $\mathrm{al}^{20}$ found success rates of $76.1 \%$ and $22.4 \%$ following trabeculectomy with MMC and AIT, respectively, at 2 years $(P<0.001)$. However, postoperative complications other than hyphema and early IOP spikes, including hypotony, wound leak, and shallow anterior chamber, occurred only in the trabeculectomy group. A prospective case series compared results from AIT-phaco with those of phaco-trab. No patients developed hypotony (IOP $<5 \mathrm{mmHg}$ ) on the first postoperative day or at the first postoperative month after AIT-phaco $(n=89)$, while three of $23(13 \%)$ eyes and four of $23(17 \%)$ eyes developed hypotony at those time points, respectively, after phaco-trab. ${ }^{17}$

\section{Clinical applications}

At its inception, AIT was indicated for adult POAG. Over the past decade, smaller trials have provided evidence supporting the use of AIT in various secondary open-angle glaucomas as well as in eyes with narrow angles. Subgroup analysis of these subtypes of glaucoma was not possible in the meta-analysis by Kaplowitz et $\mathrm{al}^{13}$ due to a limited number of patients. Findings from these smaller studies, which are largely retrospective, are described.

Glaucoma associated with inflammation can be refractory to medical therapy and often necessitates surgical intervention. Trabeculectomy is associated with higher rates of failure in eyes prone to inflammation due to scarring of the scleral flap. Tube shunt surgery is an alternative in uveitic eyes, but, like trabeculectomy, involves incising the conjunctiva and creating a full-thickness sclerostomy. Several small retrospective studies have examined the use of AIT in this patient population and suggest that AIT is effective in lowering IOP in these patients. ${ }^{36-38}$ AIT was also shown to achieve IOP control after severe IOP spikes associated with intraocular lymphoma treatment. ${ }^{39}$

Cataract surgery with intraocular lens implantation has been shown to reduce the dispersion of pseudoexfoliation material and pigment. ${ }^{40}$ AIT combined with phacoemulsification reduced IOP more than trabecular aspiration combined with phacoemulsification $(P=0.019)$ at 1 year in patients with PXG. ${ }^{41}$

IOP reduction to the mid-teens after AIT has been demonstrated in patients with PXG and pigmentary glaucoma using data from the Trabectome Study Group database. ${ }^{11,42}$ One-year success (ie, IOP $<21 \mathrm{mmHg}$ and $>20 \%$ reduction from baseline) was $79.1 \%$ and $86.7 \%$ in patients with PXG who underwent AIT alone and AIT combined with phacoemulsification, respectively, and $92 \%$ in patients with pigmentary glaucoma who underwent AIT alone or in combination with phacoemulsification.

The use of AIT in eyes with elevated episcleral venous pressure, active neovascularization, and angle dysgenesis has not been reported. AIT for childhood glaucoma has also not been studied.

The role of AIT has expanded to include eyes with narrow angles. Bussel et $\mathrm{al}^{43}$ found no statistically significant difference in IOP reduction, number of medications, success rates, or complications among patients with narrow and open angles $(P>0.05)$.

AIT was shown to be similarly effective in reducing IOP in eyes with and without prior laser trabeculoplasty (LTP) in a retrospective study using data from the Trabectome 
Study Group database. Mean \pm SD IOP was reduced from a baseline of $23.4 \pm 7.8 \mathrm{mmHg}(\mathrm{n}=852)$ to $15.8 \pm 2.7 \mathrm{mmHg}$ $(n=47)$ in eyes without prior LTP and from a baseline of $23.5 \pm 7.4 \mathrm{mmHg}(\mathrm{n}=493)$ to $14.3 \pm 2.9 \mathrm{mmHg}(n=3)$ in eyes with prior LTP at 3 years. The type of LTP and interval between LTP and subsequent AIT were not reported. ${ }^{44}$

AIT has also been evaluated as a secondary procedure following failed traditional glaucoma surgery. In a prospective study of eyes that underwent AIT or AIT-phaco at least 3 months after failed trabeculectomy using data from the Trabectome Study Group database, mean \pm SD IOP and number of medications were reduced from a baseline of $23.7 \pm 5.5 \mathrm{mmHg}$ and $2.8 \pm 1.2(\mathrm{n}=73)$ to $16.2 \pm 3.9 \mathrm{mmHg}$

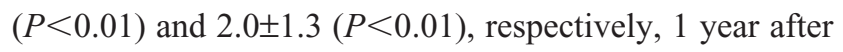
AIT alone $(n=58)$. Mean \pm SD IOP and number of medications were reduced from a baseline of $20.0 \pm 5.9 \mathrm{mmHg}$ and $2.5 \pm 1.5$ to $15.6 \pm 5.1 \mathrm{mmHg}(P=0.11)$ and $1.6 \pm 1.4(P=0.24)$, respectively, 1 year after AIT-phaco $(n=15)$. One-year success (ie, IOP $<21 \mathrm{mmHg}$ and $>20 \%$ reduction from baseline without additional glaucoma surgery) was $81 \%$ and $87 \%$ for AIT and AIT-phaco, respectively. Five (7\%) eyes developed transient hypotony (IOP $<5 \mathrm{mmHg}$ ) that resolved spontaneously without visual sequelae. Thirteen $(18 \%)$ eyes required additional glaucoma surgery. ${ }^{45}$

A similar study, also using data from the Trabectome Study Group database, examined the use of AIT in patients after failed tube shunt surgery. Mean \pm SD baseline IOP was $23.7 \pm 6.4 \mathrm{mmHg}$ using $3.2 \pm 1.5$ medications $(\mathrm{n}=20)$ and was reduced to $15.5 \pm 3.2 \mathrm{mmHg}(P=0.05)$ using $2.4 \pm 1.5$ medications $(P=0.44)$, respectively, at 1 year $(n=15)$. Success (ie, IOP $\leq 21 \mathrm{mmHg}$ and $\geq 20 \%$ reduction from baseline without additional glaucoma surgery) was $84 \%$ at 1 year. Transient hypotony (IOP $<5 \mathrm{mmHg}$ ) developed on the first postoperative day in two $(10 \%)$ eyes and three $(15 \%)$ eyes required additional glaucoma surgery. ${ }^{46}$

These two studies suggest a possible role for AIT and phaco-AIT as an alternative to further incisional surgery or cyclodestructive procedures once an initial incisional surgery has failed. However, both studies were limited by small sample size and short duration of follow-up.

\section{Cost effectiveness}

Studies of the cost effectiveness of AIT and AIT-phaco have been performed only in Canada to date. AIT was associated with cost savings of CAD \$279.23, \$1,572.55, and \$2,424.71 per patient when compared with monodrug, bidrug, and tridrug therapy, respectively, after 6 years. ${ }^{47}$

\section{Discussion}

Candidates for AIT and AIT-phaco are generally those in whom a postoperative IOP above episcleral venous pressure is sufficient to halt glaucomatous progression. Unlike incisional glaucoma surgery, AIT does not bypass downstream resistance to flow. AIT is a reasonable option to consider as a first surgery in patients with mild-to-moderate glaucoma, including those who have failed LTP. Patients with coexistent visually significant cataracts may benefit from combined AIT-phaco as an initial glaucoma operation, as it spares the conjunctiva should later filtering surgery be required.

A large body of evidence supporting the safety of AIT alone or combined with cataract surgery exists, with a very low rate of attendant complications. Hyphema, peripheral anterior synechiae, and transient IOP spikes amenable to medical therapy have been most often described. AIT is associated with modest efficacy in lowering IOP to the midto-high-teens, and some studies suggest it may have a role in relieving medication burden.

However, most data come from prospective case series and retrospective studies limited by small sample size, short duration of follow-up, and high attrition rates. Many retrospective studies have used data from the Trabectome Study Group database, ${ }^{11,15,42,44-46}$ a compendium of data from the first 20 AIT cases performed by surgeons who voluntarily provide deidentified information about their cases. The database includes a wide spectrum of diagnoses and disease severity and is prone to bias, as not every surgeon who performs AIT is obligated to share his or her experience. Furthermore, as with any relatively new surgical procedure, there is likely a "learning curve" effect that could influence surgical outcomes. Randomized, controlled trials comparing AIT-phaco with phacoemulsification cataract surgery, and comparing AIT head-to-head with other MIGS procedures, are needed to better evaluate its role in the surgical management of glaucoma.

\section{Disclosure}

The authors report no conflicts of interest in this work.

\section{References}

1. Saheb H, Ahmed II. Micro-invasive glaucoma surgery: current perspectives and future directions. Curr Opin Ophthalmol. 2012; 23(2):96-104.

2. Francis BA, Singh K, Lin SC, et al. Novel glaucoma procedures: a report by the American Academy of Ophthalmology. Ophthalmology. 2011; 118(7):1466-1480.

3. Mosaed S. The first decade of global Trabectome outcomes. Eur Ophthalmic Rev. 2014;8(2):113-119. 
4. Kaplowitz K, Schuman JS, Loewen NA. Techniques and outcomes of minimally invasive trabecular ablation and bypass surgery. $\mathrm{Br} J$ Ophthalmol. 2014;98(5):579-585.

5. Polat J, Loewen NA. Combined phacoemulsification and Trabectome for treatment of glaucoma. Surv Ophthalmolol. Epub 2016 May 6.

6. Francis BA, See RF, Rao NA, Minckler DS, Baerveldt G. Ab interno trabeculectomy: development of a novel device (Trabectome) and surgery for open angle glaucoma. J Glaucoma. 2006;15(1): 68-73.

7. Minckler D, Baerveldt G, Ramirez MA, et al. Clinical results with the Trabectome, a novel surgical device for treatment of open-angle glaucoma. Trans Am Ophthalmol Soc. 2006;104:40-50.

8. Maeda M, Watanabe M, Ichikawa K. Evaluation of Trabectome in open-angle glaucoma. J Glaucoma. 2013;22(3):205-208.

9. Werth JP, Gesser C, Klemm M. Diverse effectiveness of the Trabectome for different types of glaucoma. Klin Monbl Augenheilkd. 2015;232(1):72-78.

10. Lee JW, Yick DW, Tsang S, Yuen CY, Lai JS. Efficacy and safety of Trabectome surgery in Chinese open-angle glaucoma. Medicine (Baltimore). 2016;95(15):e3212.

11. Ting JL, Damji KF, Stiles MC; Trabectome Study Group. Ab interno trabeculectomy: outcomes in exfoliation versus primary open-angle glaucoma. J Cataract Refract Surg. 2012;38(2):315-323.

12. Mizoguchi T, Nishigaki S, Sato T, Wakiyama H, Ogino N. Clinical results of trabectome surgery for open-angle glaucoma. Clin Ophthalmol. 2015;9:1889-1894.

13. Kaplowitz K, Bussel II, Honkanen R, Schuman JS, Loewen NA. Review and meta-analysis of ab-interno trabeculectomy outcomes Br J Ophthalmol. 2016;100(5):594-600.

14. Luebke J, Boehringer D, Neuburger M, et al. Refractive and visual outcomes after combined cataract and Trabectome surgery: a report on the possible influences of combining cataract and Trabectome surgery on refractive and visual outcomes. Graefes Arch Clin Exp Ophthalmol. 2015;253(3):419-423.

15. Francis BA, Minckler D, Dustin L, et al; Trabectome Study Group. Combined cataract extraction and trabeculotomy by the internal approach for coexisting cataract and open-angle glaucoma: initial results. J Cataract Refract Surg. 2008;34(7):1096-1103.

16. Francis B. Trabectome combined with phacoemulsification versus phacoemulsification alone: a prospective, nonrandomized controlled surgical trial. Clin Surg Ophthalmol. 2010;28:1-7.

17. Francis B, Winarko J. Combined Trabectome and cataract surgery versus combined trabeculectomy and cataract surgery in open-angle glaucoma. Clin Surg Ophthalmol. 2011;29:4-10.

18. Parikh HA, Bussel II, Schuman JS, Brown EN, Loewen NA. Coarsened exact matching of phaco-Trabectome to Trabectome in phakic patients: lack of additional pressure reduction from phacoemulsification. PLoS One. 2016;11(2):e0149384.

19. Fellman RL, Feuer WJ, Grover DS. Episcleral venous fluid wave correlates with Trabectome outcomes: intraoperative evaluation of the trabecular outflow pathway. Ophthalmology. 2015;122(12): 2385.e1-2391.e1.

20. Jea SY, Francis BA, Vakili G, Filippopoulos T, Rhee DJ. Ab interno trabeculectomy versus trabeculectomy for open-angle glaucoma. Ophthalmology. 2012;119(1):36-42.

21. Jea SY, Mosaed S, Vold SD, Rhee DJ. Effect of a failed Trabectome on subsequent trabeculectomy. J Glaucoma. 2012;21(2): 71-75.

22. Gedde SJ, Herndon LW, Brandt JD, et al; Tube Versus Trabeculectomy Study Group. Postoperative complications in the Tube Versus Trabeculectomy (TVT) study during five years of follow-up. Am J Ophthalmol. 2012;153(5):804.e1-814.e1.

23. Christakis PG, Tsai JC, Kalenak JW, et al; The Ahmed versus Baerveldt study: three-year treatment outcomes. Ophthalmology. 2013;120(11): 2232-2240.
24. Budenz DL, Feuer WJ, Barton K, et al; Ahmed Baerveldt Comparison Study Group. Postoperative complications in the Ahmed Baerveldt Comparison Study during five years of follow-up. Am J Ophthalmol. 2016;163:75.e3-82.e3.

25. Weiner Y, Severson ML, Weiner A. Intraocular pressure 3 to 4 hours and 20 hours after cataract surgery with and without ab interno trabeculectomy. J Cataract Refract Surg. 2015;41(10):2081-2091.

26. Ahuja Y, Malihi M, Sit AJ. Delayed-onset symptomatic hyphema after ab interno trabeculotomy surgery. Am J Ophthalmol. 2012;154(3): 476.e2-480.e2.

27. Knape RM, Smith MF. Anterior chamber blood reflux during trabeculectomy in an eye with previous Trabectome surgery. J Glaucoma. 2010;19(7):499-500.

28. Parekh AS, Weinreb RN, Dorairaj SK. Delayed-onset symptomatic hyphema after ab interno trabeculotomy surgery. Am J Ophthalmol. 2013;155(4):778-779.

29. Mansouri K, Medeiros FA, Weinreb RN. Twenty-four-hour intraocular pressure patterns in a symptomatic patient after ab interno trabeculotomy surgery. Clin Ophthalmol. 2014;8:2195-2197.

30. Minckler DS, Baerveldt G, Alfaro MR, Francis BA. Clinical results with the Trabectome for treatment of open-angle glaucoma. Ophthalmology. 2005;112(6):962-967.

31. Francis BA, Winarko J. Ab interno Schlemm's canal surgery: trabectome and I-stent. Dev Ophthalmol. 2012;50:125-136.

32. Akagi T, Nakano E, Nakanishi H, Uji A, Yoshimura N. Transient ciliochoroidal detachment after ab interno trabeculotomy for open-angle glaucoma: a prospective anterior-segment optical coherence tomography study. JAMA Ophthalmol. 2016;134(3):304-311.

33. Osman EA, AlMobarak F. Ciliochoroidal effusion with persistent hypotony after Trabectome surgery. Indian J Ophthalmol. 2015;63(3): 272-274.

34. Jordan JF, Wecker T, van Oterendorp C, et al. Trabectome surgery for primary and secondary open angle glaucomas. Graefes Arch Clin Exp Ophthalmol. 2013;251(12):2753-2760.

35. Mosaed S, Rhee D, Filippopoulos T, Tseng H, Deokule S, Weinreb RN. Trabectome outcomes in adult open-angle glaucoma patients: one-year follow-up. Clin Surg Ophthalmol. 2010;28:5-9.

36. Anton A, Heinzelmann $\mathrm{S}, \mathrm{Ne} ß \mathrm{~T}$, et al. Trabeculectomy ab interno with the Trabectome ${ }^{\circledR}$ as a therapeutic option for uveitic secondary glaucoma. Graefes Arch Clin Exp Ophthalmol. 2015;253(11): 1973-1978.

37. Pahlitzsch M, Torun N, Gonnermann J, et al. Trabeculectomy ab interno (Trabectome): yet another possibility in the treatment of uncontrolled glaucomatocyclitic crisis under systemic valganciclovir therapy? Eye (Lond). 2015;29(10):1335-1339.

38. Shimizu A, Maruyama K, Yokoyama Y, Tsuda S, Ryu M, Nakazawa T. Characteristics of uveitic glaucoma and evaluation of its surgical treatment. Clin Ophthalmol. 2014;8:2383-2389.

39. van Oterendorp C, Ness T, Illerhaus G, Neuburger M, Jordan JF. The Trabectome as treatment option in secondary glaucoma due to intraocular lymphoma. J Glaucoma. 2014;23(7):482-484.

40. Jacobi PC, Dietlein TS, Krieglstein GK. Bimanual trabecular aspiration in pseudoexfoliation glaucoma: an alternative in nonfiltering glaucoma surgery. Ophthalmology. 1998;105(5):886-894.

41. Klamann MK, Gonnermann J, Maier AK, et al. Combined clear cornea phacoemulsification in the treatment of pseudoexfoliative glaucoma associated with cataract: significance of trabecular aspiration and ab interno trabeculectomy. Graefes Arch Clin Exp Ophthalmol. 2013; 251(9):2195-2199.

42. Akil H, Chopra V, Huang A, Loewen N, Noguchi J, Francis BA. Clinical results of $\mathrm{ab}$ interno trabeculotomy using the Trabectome in patients with pigmentary glaucoma compared to primary open angle glaucoma. Clin Experiment Ophthalmol. Epub 2016 Mar 6.

43. Bussel II, Kaplowitz K, Schuman JS, Loewen NA; Trabectome Study Group. Outcomes of ab interno trabeculectomy with the Trabectome by degree of angle opening. Br J Ophthalmol. 2015;99(7):914-919. 
44. Vold SD, Dustin L; Trabectome Study Group. Impact of laser trabeculoplasty on Trabectome ${ }^{\circledR}$ outcomes. Ophthalmic Surg Lasers Imaging. 2010;41(4):443-451.

45. Bussel II, Kaplowitz K, Schuman JS, Loewen NA; Trabectome Study Group. Outcomes of ab interno trabeculectomy with the Trabectome after failed trabeculectomy. Br J Ophthalmol. 2015;99(2): $258-262$.
46. Mosaed S, Chak G, Haider A, Lin KY, Minckler DS. Results of Trabectome surgery following failed glaucoma tube shunt implantation: cohort study. Medicine (Baltimore). 2015;94(30):e1045.

47. Iordanous Y, Kent JS, Hutnik CM, Malvankar-Mehta MS. Projected cost comparison of Trabectome, iStent, and endoscopic cyclophotocoagulation versus glaucoma medication in the Ontario Health Insurance Plan. J Glaucoma. 2014;23(2):e112-e118.

\section{Publish your work in this journal}

Clinical Ophthalmology is an international, peer-reviewed journal covering all subspecialties within ophthalmology. Key topics include: Optometry; Visual science; Pharmacology and drug therapy in eye diseases; Basic Sciences; Primary and Secondary eye care; Patient Safety and Quality of Care Improvements. This journal is indexed on

\section{Dovepress}

PubMed Central and CAS, and is the official journal of The Society of Clinical Ophthalmology (SCO). The manuscript management system is completely online and includes a very quick and fair peer-review system, which is all easy to use. Visit http://www.dovepress.com/ testimonials.php to read real quotes from published authors. 